\title{
Circuit weight training in early cardiac rehabilitation
}

\author{
RICHARD M. BUTLER, DO \\ GEORGIANNE PALMER, BS \\ FELIX J. ROGERS, DO
}

A prospective, randomized study evaluated the feasibility, safety, and efficacy of upper body circuit weight training (CWT) in 25 stable male cardiac patients entering the initial out-of-hospital phase of cardiac rehabilitation. Both groups performed 30 minutes of aerobic exercise only for 6 weeks. The aerobic exercise group $(N=13)$ continued this regimen for 6 more weeks, during which time the CWT group $(\mathrm{N}=12)$ performed $15 \mathrm{~min}$ utes of aerobic exercise followed by CWT (two loops, eight upper body exercises). The only adverse response was in one CWT patient in whom restenosis developed. Peak heart rate during aerobic exercise and CWT was similar, but peak systolic blood pressure during aerobic exercise was significantly greater than during CWT. Peak rate pressure product during aerobic exercise and CWT was similar. Treadmill time increased significantly in both groups. Upper body strength (cumulative pounds lifted) increased significantly only in the CWT group. A coordinated program of CWT and aerobic exercise can be performed safely in stable cardiac patients during phase 2 cardiac

From the Department of Medicine, Wayne State University, School of Medicine, Detroit, Mich (Dr Butler), and the Division of Cardiology, Riverside Osteopathic Hospital, Trenton, Mich (Dr Rogers).

Supported by the Horizon Health System's Grant-inAid program, Southfield, Mich.

Reprint requests to Richard M. Butler, DO, University Health Center, 4201 St Antoine, Detroit, MI 48201. rehabilitation, resulting in improved upper body strength and aerobic capacity.

(Key words: weight lifting, heart, rehabilitation, exercise therapy, coronary artery disease)

Traditionally, cardiac rehabilitation programs have emphasized aerobic activities such as walking, jogging, and cycling to promote cardiovascular fitness. These exercises focus primarily on the lower extremities and may not improve upper body strength and endurance. However, most cardiac patients daily perform many activities that require upper body strength and endurance. In addition, numerous cardiac patients are employed in jobs that require upper body activities.

Recently, the hemodynamic responses in cardiac patients performing upper body resistance exercises against moderate loads (30\% to $40 \%$ of the maximal voluntary contraction) were evaluated. ${ }^{1-4}$ Cardiac ischemia and adverse increases in heart rate and blood pressure were not observed. One form of resistance exercise that has been incorporated into cardiac rehabilitation programs is circuit weight training (CWT). ${ }^{5}$ Three prospective studies evaluating the use of CWT in cardiac rehabilitation programs for periods of 10 weeks to 3 years have documented improvements in upper body strength in clinically stable cardiac patients. ${ }^{2,6,7}$ That adverse clinical responses were not observed indicates the safety of resistance exercises in stable cardiac patients. However, these studies evaluated only patients who had 
been enrolled in a cardiac rehabilitation program for a minimum of 3 months before beginning CWT.

Recent guidelines for the use of strength training exercises with cardiac patients suggest that patients be regular participants in a cardiac rehabilitation program for at least 12 weeks before beginning CWT. ${ }^{8,9}$ However, most cardiac patients return to work in 8 to 12 weeks after a cardiac event. ${ }^{10}$ Therefore, if strength training exercises are to be used to promote full recovery of cardiac patients and ensure safe return to work, then their use in earlier phases of cardiac rehabilitation must be evaluated.

To our knowledge, the use of upper body CWT in the initial out-of-hospital phase of cardiac rehabilitation (phase 2), before most cardiac patients have returned to work, has not been reported. The purpose of this study was to evaluate the feasibility, safety, and efficacy of performing a coordinated program of upper body CWT and standard aerobic exercise within the setting of a community-based, 12week, phase 2 cardiac rehabilitation program. Cardiovascular responses and changes in strength and aerobic capacity in male cardiac patients performing a combined program of aerobic exercise plus upper body CWT were compared with those of patients performing a traditional program of aerobic exercise only.

\section{Methods}

Male cardiac patients entering a phase 2 cardiac rehabilitation program were evaluated consecutively for possible enrollment into the study. A general history was taken with review of cardiac procedures and medications and a physican examination was performed on all patients. Patients were excluded from enrollment if the following criteria were present: age greater than 65 years, unstable angina pectoris, uncontrolled hypertension (systolic blood pressure $>160 \mathrm{~mm} \mathrm{Hg}$ or diastolic blood pressure $>100 \mathrm{~mm} \mathrm{Hg}$ ), symptomatic dysrhythmias, or congestive heart failure. Twenty-five male patients aged $52.5 \pm 8.5$ years $($ mean \pm 1 standard deviation [SD]) gave informed consent and were enrolled in the study. Patients were assigned to either an aerobic exercise group $(n=13)$ or a CWT group $(\mathrm{n}=12$ ) by means of a randomized block design to match for a history of revascularization pro- cedures, myocardial infarction, and use of $\beta$-adrenergic blocking agents.

\section{Exercise protocol}

The phase 2 cardiac rehabilitation program was 12 weeks in duration with three exercise sessions per week.

Aerobic exercise-Aerobic exercise was performed by either cycling on a Schwinn Airdyne cycle (Schwinn Bicycle Co, Chicago, Ill) or walking on a Quinton 18-56 treadmill (Quinton Medical Instrument Co, Seattle, Wash). The training heart rate during aerobic exercise was set by the exercise therapist in a range of either $70 \%$ to $85 \%$ of maximum heart rate or $65 \%$ to $80 \%$ of heart rate reserve (calculated by subtracting resisting heart rate from maximum heart rate). Maximum heart rate was measured during sign- and symptomlimited exercise testing performed to assess initial measures of aerobic fitness, to be described later.

All patients underwent measurement of maximum heart rate within 2 weeks of enrollment into the study. For patients who had not completed exercise testing before beginning the exercise protocol, training heart rate was set at resting heart rate plus 30 ; the exercise prescription was adjusted when measured maximum heart rate was obtained. A single-lead telemetry monitor was worn during aerobic exercise to ensure that patients stayed within the prescribed heart rate range.

During the first 6 weeks of training, all patients in both the aerobic exercise group and the CWT group performed the same program of aerobic exercise only. Each exercise session began with $5 \mathrm{~min}$ utes of low-intensity aerobic exercise to allow for muscle warm-up, immediately followed by $30 \mathrm{~min}$ utes of continuous exercise in the training heart rate range. A 2-minute cool-down period of lowintensity exercise concluded each session. The total 37 minutes of aerobic exercise was performed on one piece of equipment. Because of limited availability of treadmills in the phase 2 program, approximately $75 \%$ of the aerobic exercise was performed on the Schwinn Airdyne cycle, with the remaining $25 \%$ on the treadmill.

Circuit weight training-In the sixth week of training, all patients in both the aerobic exercise group and the CWT group were instructed on the use of the circuit weight training equipment, including proper body positioning and breathing during exertions. The CWT was performed on Keiser Cam II pneumatic resistance machines (Keiser Corp, Fresno, Calif). This equipment uses air-filled cylinders to provide a variable pneumatic resis- 
tance measured in pounds per square inch (psi) throughout a full range of motion. The weighttraining "circuit" consisted of eight independent stations each exercising a different upper body muscle group (triceps, upper back, lateral pull-down, chest press, arm curl, butterfly, shoulder raise, and military press).

After each patient demonstrated proper technique on the equipment at low levels of resistance, we then determined the maximum resistance against which a single exertion could be completed at each station in the circuit. To measure maximum resistance, patients performed a series of single movements against incremental increases in resistance until an increase of less than 5 psi prevented the completion of the next single movement. The resistance during the last complete movement was labeled the one-movement maximum. The procedure was repeated for each station in the CWT loop.

Patients were closely monitored and coached to exhale during the exertions to avoid breath holding and grunting. Each movement had to be continuous to avoid isometric contraction. If an exertion could not be completed because of excessive resistance, then the patient was instructed to relax immediately. One-minute rest periods were used between exertions. We were able to determine a patient's one-movement maximum at each station using no more than four exertions.

After the initial determination of the one-movement maximums, patients were informed of their group assignment. During the weeks 7 through 12 of the training protocol, patients in the aerobic exercise group completed training by performing the same program of 37 minutes of continuous aerobic exercise as described for the first 6 weeks.

In contrast, during the final 6 weeks, patients in the CWT group performed a combined program of aerobic exercise and CWT. Each exercise session began with 5 minutes of low-intensity aerobic exercise followed immediately by 15 minutes of continuous aerobic exercise in the training heart rate range. Aerobic exercise was performed on the cycle or the treadmill during $75 \%$ and $25 \%$ of the remaining exercise sessions, respectively. Only one piece of equipment was used for aerobic exercise during each session. At the completion of $15 \mathrm{~min}$ utes of aerobic exercise at training heart rate, the patients moved directly to the CWT loop.

During the CWT portion of exercise, patients performed ten repetitions at each station against a resistance set at $40 \%$ of the measured one-movement maximum. The patients moved through the circuit with 40 seconds of rest between stations. Two loops ( 16 stations) were completed during each session in approximately 15 to 17 minutes.

Patients in the CWT group were retested for onemovement maximum at each exercise station early in weeks 9 and 11 to evaluate for increases in strength. Resistance during subsequent training was increased as necessary to maintain the exercise intensity at $40 \%$ of the new one-movement maximum.

\section{Patient monitoring}

A certified, cardiac exercise therapist was present during all exercise sessions to monitor patient responses and to assure patient safety. During CWT, the therapist helped set the exercise resistance and timed rest periods between machines. A physician was readily available during all sessions.

Continuous electrocardiographic responses were monitored with a telemetry monitor using a modified $V_{5}$ lead during all sessions of aerobic exercise, CWT, and strength testing. Heart rate was obtained from numerical values appearing on the telemetry screen. During each aerobic exercise session, the following heart rate values were recorded into the patient's chart: at rest, midway through the exercise period with the patient at training heart rate, and 5 minutes after completing exercise. During each CWT session, heart rate values were recorded into the patient's chart as follows: immediately on completion of the tenth repetition at each station (peak heart rate for that station), and 5 minutes after the patient completed the two circuits.

Blood pressure was measured with a calibrated aneroid sphygmomanometer and blood pressure cuff with auscultation over the brachial artery. Although blood pressure was checked in both arms, the arm with a higher value was used. During each aerobic exercise session, blood pressure was recorded at rest, midway through the exercise period with the patient at training heart rate, and 5 minutes after completing exercise. During CWT, blood pressure measurements were obtained during a single exercise session in weeks 6,9 , and 12 . To facilitate prompt measurement, the patient exercised at each station with the blood pressure cuff deflated and taped in place on the forearm. The cuff was inflated during the final contraction at each station in the two loops. Immediately on the completion of the final contraction, a stethoscope was placed over the brachial artery and the cuff was deflated to allow for the assessment of blood pressure. 


\section{Outcome measures}

Measures of aerobic capacity were assessed with treadmill exercise testing limited by signs and symptoms according to the standard Bruce protocol. ${ }^{11}$ Continuous electrocardiographic responses were monitored throughout each test. Twelve-lead electrocardiographic responses and cuff occlusion blood pressure were recorded at rest in the supine, seated, and standing position immediately before the test and during each stage of the test. Aerobic capacity was estimated from total time on the treadmill to maximal effort in seconds. Patients were coached throughout the test to assure that no one used the support bars at any time and to encourage each patient to continue the test to maximal effort.

All patients were scheduled to undergo initial measures of aerobic capacity within 2 weeks before starting exercise. However, because of scheduling conflicts, initial exercise testing was performed on seven patients in the aerobic exercise group 2 to 14 days after starting training and six patients in the CWT group 8 to 14 days after starting training. Final measures of aerobic fitness were obtained within 0 to 12 days and 3 to 12 days of completing the 12-week protocol in the aerobic exercise and CWT groups, respectively.

Measures of strength were assessed by calculating the arithmetic sum of weight lifted during the one-movement maximum exertions performed at each of the eight stations in the CWT loop. Initial measures of strength were obtained on all patients during week 6 of the exercise protocol. Final measures of strength were obtained $2 \pm 1$ and $1 \pm 1$ (mean $\pm \mathrm{SD}$ ) days after completing the 12-week protocol on patients in the aerobic exercise and CWT group, respectively.

\section{Statistical analysis}

All units of exercise resistance on the CWT apparatus were normalized by converting pounds per square inch to units of "pounds lifted" with tables supplied by the Keiser Corporation. fifl Analysis and two-tailed Student's $t$ test were used to assess differences within and between groups as appropriate. Differences were considered significant if the $P$ value was $<.05$. All values are reported as mean \pm 1 standard error (SE) unless otherwise noted.

\section{Results}

At entry, the aerobic exercise group $(n=13)$ and the CWT group $(\mathrm{n}=12)$ had a similar mean age $(53 \pm 2$ and $51 \pm 2$ years, respectively), initial treadmill duration $(390 \pm 22$ and $449 \pm 27$ seconds, respectively), and initial strength $(735 \pm 63$ and $742 \pm 31$ cumulative pounds lifted, respectively). The clinical characteristics of each group are presented in Table $1 . \chi^{2}$ Analysis indicated that the clinical characteristics of the two groups were not significantly different.

\section{Completion of exercise protocol}

All patients in both groups completed the first 6 weeks of exercise training and initial strength testing without the development of chest pain, complex ventricular ectopy, paroxaysmal supraventricular tachycardia, or ischemic ST segment responses on telemetry recordings. All 13 patients in the aerobic exercise group completed the final 6 weeks of exercise without abnormal responses.

In the CWT group, recurrent chest pain developed during week 7 in one patient who had undergone single-vessel coronary angioplasty before enrollment, so he was removed from the study. Repeat angiography revealed restenosis of his left anterior descending artery. The remaining 11 patients in the CWT group completed the final 6 weeks of aerobic exercise plus CWT without having chest pain, complex ventricular ectopy, paroxysmal supraventricular tachycardia, or ischemic ST segment responses.

The total number of exercise sessions completed by the aerobic exercise group $(n=13)$ and the CWT group $(n=11)$ during the 12 weeks was similar ( $30 \pm 1$ and $32 \pm 1$, respectively). The CWT group performed $17 \pm 1$ sessions of combined aerobic and resistive exercise during weeks 7 through 12 of the protocol.

\section{Hemodynamic responses to CWT}

A within-group analysis was performed on patients in the CWT group to compare the hemodynamic responses during CWT to responses during aerobic exercise. Peak heart rate and blood pressure responses during CWT and aerobic exercise were compared during a single exercise session at the end of week 9 , midway through the final 6 weeks of training (Table 2). Mean peak heart rate during CWT and aerobic exercise was similar. However, mean peak 


\begin{tabular}{|c|c|c|}
\hline \multicolumn{3}{|c|}{$\begin{array}{l}\text { Table 1 } \\
\text { Clinical Characteristics of Cardiac Patients in the } \\
\text { Aerobic Exercise and Circuit Weight Training Groups* }\end{array}$} \\
\hline \multirow[b]{2}{*}{ Characteristic } & \multicolumn{2}{|c|}{ Group } \\
\hline & $\begin{array}{c}\text { Aerobic } \\
\text { exercise } \\
(\mathrm{N}=13)\end{array}$ & $\begin{array}{c}\text { Circuit } \\
\text { weight } \\
\text { training } \\
(\mathrm{N}=12)\end{array}$ \\
\hline $\begin{array}{l}\text { Myocardial infarction } \\
\text { - None } \\
\text { - Anterior wall } \\
\text { - Inferior wall }\end{array}$ & $\begin{array}{l}5 \\
3 \\
5\end{array}$ & $\begin{array}{l}6 \\
3 \\
3\end{array}$ \\
\hline $\begin{array}{l}\text { Revascularization } \\
\text { procedures } \\
\text { - None } \\
\text { - Coronary artery bypass graft } \\
\text { Percutaneous transluminal } \\
\text { coronary angioplasty }\end{array}$ & $\begin{array}{l}5 \\
5 \\
3\end{array}$ & $\begin{array}{l}3 \\
4 \\
5\end{array}$ \\
\hline $\begin{array}{l}\text { No. of coronary vessels with } \\
>\mathbf{5 0} \% \text { luminal occlusion } \\
\text { 1 Vessel } \\
\text { 2 Vessels } \\
\text { - } 3 \text { Vessels }\end{array}$ & $\begin{array}{l}6 \\
3 \\
4\end{array}$ & $\begin{array}{l}9 \\
2 \\
1\end{array}$ \\
\hline$\beta$-Blockers & 6 & 4 \\
\hline
\end{tabular}

Table 2

Hemodynamic Responses* of Circuit Weight Training (CWT) Group $(n=11)$ to CWT and Aerobic Exercise During Week 9

\begin{tabular}{|llcc|}
\hline Hemodynamic variable & $\begin{array}{c}\text { Circuit } \\
\text { weight } \\
\text { training }\end{array}$ & $\begin{array}{c}\text { Aerobic } \\
\text { exercise }\end{array}$ & $\begin{array}{c}\text { Statistical } \\
\text { significance }\end{array}$ \\
\hline $\begin{array}{l}\text { - Peak heart rate, } \\
\text { beats per minute }\end{array}$ & $117 \pm 7$ & $112 \pm 6$ & NS \\
$\begin{array}{l}\text { - Peak systolic blood } \\
\text { pressure, mm Hg }\end{array}$ & $113 \pm 4$ & $131 \pm 4$ & $P<.005$ \\
$\begin{array}{l}\text { - Peak rate-pressure } \\
\text { product } \dagger\end{array}$ & $133 \pm 12$ & $148 \pm 11$ & NS \\
\hline $\begin{array}{l}\text { *All values are given as mean } \pm 1 \text { standard error. } \\
\text { †Rate-pressure product }=(\text { systolic blood pressure } \times \text { heart rate }) / 100 .\end{array}$ & & \\
\hline
\end{tabular}


systolic blood pressure during CWT was significantly less than the value measured during aerobic exercise. The mean peak rate-pressure product (systolic blood pressure $\times$ heart rate)/100) during CWT and aerobic exercise was similar.

\section{Aerobic capacity and strength}

Three patients in the aerobic exercise group and two patients in the CWT group returned to work immediately on completion of the 12 week training program and failed to perform final graded exercise testing. The remaining patients in the aerobic exercise group $(n=10)$ and the CWT group $(n=9)$ completed final measures of strength testing without symptoms of chest pain, sustained dysrhythmias, or episodes of ischemic ST segment responses.

Table 3 presents changes in aerobic capacity and strength within each group. Treadmill duration increased by $114 \pm 8(30 \%)$ seconds in the aerobic exercise group $(P<.01)$ and by $101 \pm 21(23 \%)$ seconds in the CWT group $(P<.001)$. Final treadmill duration and changes in treadmill duration at completion were similar in both groups. The $14 \%$ increase in cumulative weight lifted in the aerobic exercise group (94 $\pm 46 \mathrm{lb})$ did not reach statistical significance. In contrast, a $22 \%$ increase in strength was seen in the CWT group $(157 \pm 8 \mathrm{lb}, P<.001)$. At completion, comparisons between the aerobic exercise and CWT groups in the total cumulative weight lifted and changes in weight lifted were not statistically different.

\section{Discussion}

This study demonstrated that selected male cardiac patients can successfully perform a coordinated program of traditional aerobic exercise and CWT during the final 6 weeks of the initial out-of-hospital phase of cardiac rehabilitation (phase 2). Adverse cardiovascular responses did not occur during CWT. Patients performing both CWT and aerobic exercise showed significant improvements in aerobic capacity and strength. In contrast, patients who performed only aerobic exercise showed increases in aerobic capacity without significant changes in upper body strength.
In our study, the performance of both maximal strength testing and prescribed resistance training at $40 \%$ of the one-movement maximum during CWT was not associated with evidence of cardiac ischemia or dysrhythmia. The one patient in the CWT group who was removed from the study during week 7 (the first week of CWT) had experienced restenosis of a coronary artery that had been previously dilated during coronary angioplasty. Restenosis following this angioplastic procedure is a welldocumented event. ${ }^{12}$ The small number of patients in this study does not allow us to determine if CWT is a risk factor for restenosis after coronary angioplasty.

The apparent safety of both strength testing and resistance training during this study may be related to several factors. First, our CWT protocol used a lower intensity of resistive effort (ten repetitions at $40 \%$ of the onemovement maximum) and longer rest periods between stations ( 40 seconds) compared with CWT in healthy persons (12 to 15 repetitions at $40 \%$ to $60 \%$ of the one-movement maximum with 15 to 30 seconds rest between stations). ${ }^{5}$ Second, the subjects in the CWT group were stable cardiac patients with a preponderance of single-vessel coronary artery disease. Third, all patients were monitored closely to ensure that proper technique was used during CWT and that a Valsalva's maneuver was not performed during exertions.

We observed no adverse effects during strength testing when using single movements against increasing resistance to determine onemovement maximums. Our patients were closely coached during this procedure to avoid breath holding. All contractions had to be smooth, continuous movements. Our previous and ongoing experience with resistive CWT exercise in cardiac patients has convinced us that this is an efficient and safe method of determining an exercise prescription for this mode of activity.

During CWT, the mean peak heart rate was similar to that during aerobic exercise (Table 2 ). These increases are similar to those reported by other investigators evaluating cardiac patients performing CWT at similar intensities with rest periods between 30 and 60 


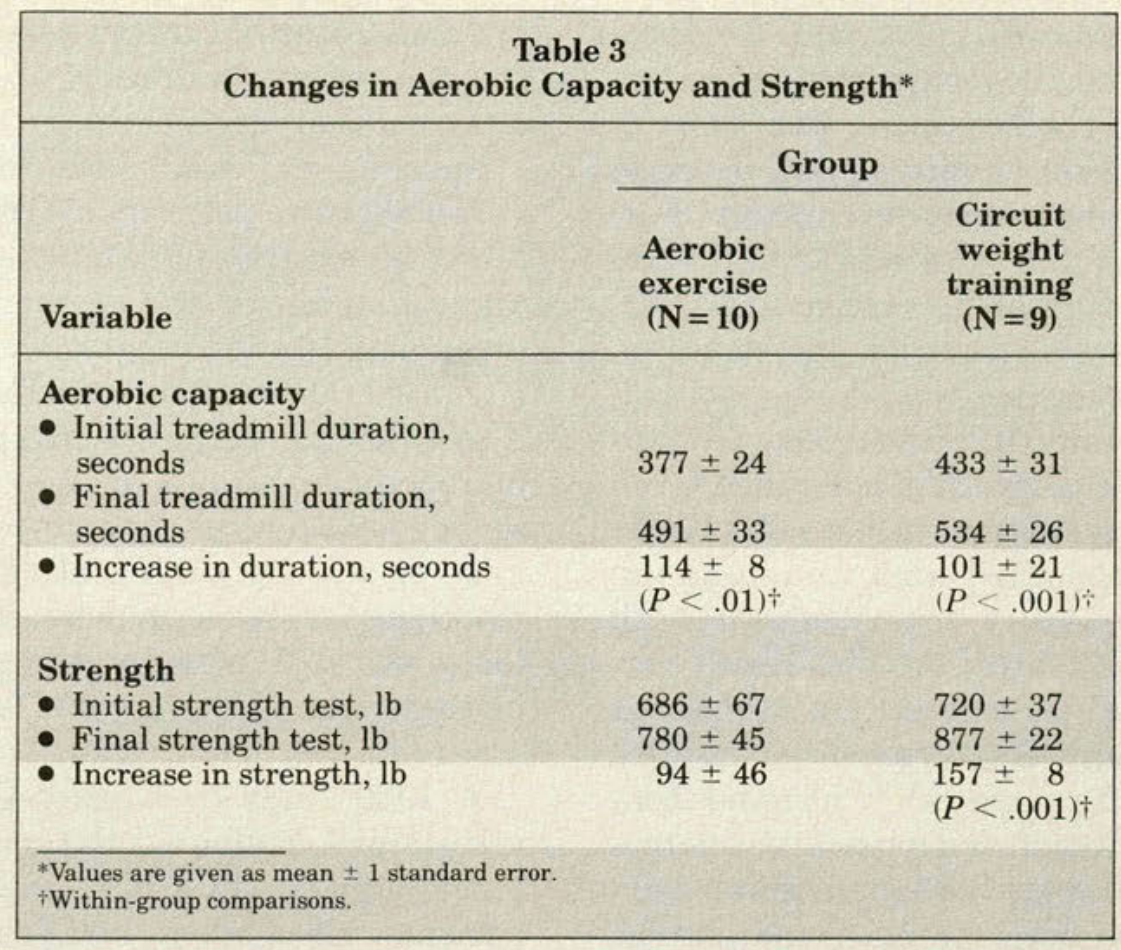

seconds. ${ }^{2,3}$ Of note is a single study by Vander and associates, ${ }^{1}$ which found only small increases in heart rate ( 3 to 7 beats per minute) in cardiac patients performing upper body CWT at $40 \%$ to $60 \%$ of maximum exertion with 10 to 20 seconds' rest between stations. Reasons for a diminished heart rate response in their patients are not clear.

Mean peak systolic blood pressure during CWT $(113 \pm 4 \mathrm{~mm} \mathrm{Hg})$ was significantly lower than the mean peak value during aerobic exercise ( $131 \pm 4 \mathrm{~mm} \mathrm{Hg}$, Table 2$)$. In our study, blood pressure was measured indirectly by cuff occlusion. Even though we attempted to measure blood pressure rapidly on the patients' completion of a final exertion, our methods may have underestimated the peak blood pressure response during CWT.

Using intra-arterial catheterization for direct measurement of blood pressure, Haslam and co-workers ${ }^{4}$ reported higher mean peak systolic blood pressure $(176 \pm 8 \mathrm{~mm} \mathrm{Hg})$ in cardiac patients performing repetitive weight lifting against a resistance of $40 \%$ maximum exertion. Investigators using invasive techniques have shown that the increases in blood pressure occurring during repetitive weight lifting fall rapidly on completion of exertion. ${ }^{13}$
Other studies using indirect measurements to assess blood pressure response during CWT found either no significant increase in systolic or diastolic blood pressure compared with resting levels ${ }^{3,7}$ or only modest increases. ${ }^{2}$

The mean peak rate-pressure product, an indirect assessment of myocardial oxygen consumption, ${ }^{14}$ was similar during CWT and aerobic exercise in our patients. This finding suggests that CWT at $40 \%$ maximum exertion re ggsulted in increases in myocardial oxygen consumption similar to those in traditionally prescribed aerobic exercise. The mean rate-pressure product during CWT of $133 \pm 12$ in our study appeared lower than the value of $167 \pm 15$ measured directly with intra-arterial catheterization during repetitive arm curls at $40 \%$ maximum exertion by Haslam and coauthors. ${ }^{4}$ However, even when invasive techniques are used for direct measurement of blood pressure, rate-pressure products during weight lifting at $40 \%$ maximum exertion appear similar to or lower than values measured during aerobic exercise at $85 \%$ of functional capacity. ${ }^{4}$ These findings all support the safety of moderate resistance training.

The patients in the CWT group had increases in treadmill duration similar to those 
of patients who performed only traditional aerobic exercise for the 12 -week-period. These results suggest that substituting two loops of CWT for half of the standard aerobic exercise does not limit gains in aerobic capacity. Few studies have evaluated the effects of a combined program of aerobic exercise and CWT on changes in aerobic capacity and strength. In a study evaluating healthy adults, Gettman and associates ${ }^{15}$ compared the effects of standard CWT with a program of combined CWT and running. The subjects in both the CWTonly group and the CWT-and-running group had similar increases in upper body strength as well as treadmill duration. This result suggests that CWT alone may have a significant aerobic component. However, other studies evaluating healthy subjects ${ }^{15-17}$ have not confirmed this observation and most investigators recommend combining CWT with aerobic exercise to improve both strength and aerobic capacity.

Kelemen and associates ${ }^{2}$ evaluated the effects of a combined program of CWT and aerobic exercise on changes in aerobic fitness and strength in male cardiac patients enrolled in a community-based cardiac rehabilitation program. Their patients had been participating in the exercise program for a minimum of 12 weeks before enrollment. During a 10 -weekperiod, those performing aerobic exercise only (20 minutes of walking/jogging at $85 \%$ of maximal heart rate plus 20 minutes of volleyball three times per week) showed no changes in treadmill duration or strength. In contrast, patients performing combined CWT (two circuits of ten stations, 10 to 15 repetitions per station at $40 \%$ of the one-movement maximum, $30 \mathrm{sec}-$ onds of rest between stations) plus aerobic exercise (20 minutes of walking/jogging at $85 \%$ maximum heart rate) three times per week increased treadmill duration by $12 \%$ and strength by $24 \%$. This $24 \%$ increase in strength was similar to the $22 \%$ increase in strength seen in our patients performing both CWT and aerobic exercise. The larger increase in treadmill duration seen in our patients (23\%) compared with those of Kelemen and associates $(12 \%)$ was most likely due to our evaluation of patients in the earliest out-of- hospital phase of cardiac rehabilitation when they lacked a minimum of 12 weeks of previous conditioning.

Sparling and co-workers ${ }^{7}$ evaluated the effect of adding a program of CWT to a program of aerobic exercise in 16 middle-aged male cardiac patients who had been enrolled in a cardiac rehabilitation program for a minimum of 3 months. The circuit consisted of 12 stations. Three times a week, patients performed 12 to 20 repetitions at $30 \%$ to $40 \%$ of the one-movement maximum at each station with $60 \mathrm{sec}-$ onds of rest between stations. During a 6month period, strength increased by $22 \%$, similar to the $22 \%$ gain in strength seen in our patients. Changes in aerobic capacity were not assessed.

A 1989 study by Crozier Ghilarducci and associates $^{18}$ evaluated the use of a more intense regimen of CWT in nine stable cardiac patients, all of whom had been enrolled in a cardiac exercise program for a minimum of 3 months. Their patients performed a combined program involving 30 minutes of stretching, aerobic exercise, and calisthenics plus $30 \mathrm{~min}$ utes of CWT three times per week. The circuit included five resistance exercises plus sit-ups. Eight to 12 repetitions at $80 \%$ maximal voluntary contraction were performed at each of the five resistance stations. Rest periods were at least 1 minute. The patients were not allowed to start the next exercise until measured blood pressure and heart rate had returned to $15 \%$ of the resting value. To determine one-movement maximum, these investigators used a protocol similar to ours. Adverse cardiovascular responses did not occur during strength testing or during training with the use of greater levels of resistance. During a 10 -week period, strength measured at each station increased from $12 \%$ to $53 \%$. Changes in aerobic capacity were not assessed. The use of higher levels of resistance resulted in greater increases in strength than seen in our patients. However, further study is needed to evaluate the safety of higher levels of resistance in phase 2 cardiac rehabilitation programs.

In our study, significant increases in strength during the final 6 weeks of training

(continued on page 89) 


\section{Get in writing}

2. Reducing the risk of coronary heart disease,

This indication can be found in the labeling of only one lipid medication 


\section{Look at the labeling}

LOPID (gemfibrozil) - the only lipid medication specifically indicated to reduce the risk of $\mathrm{CHD}$
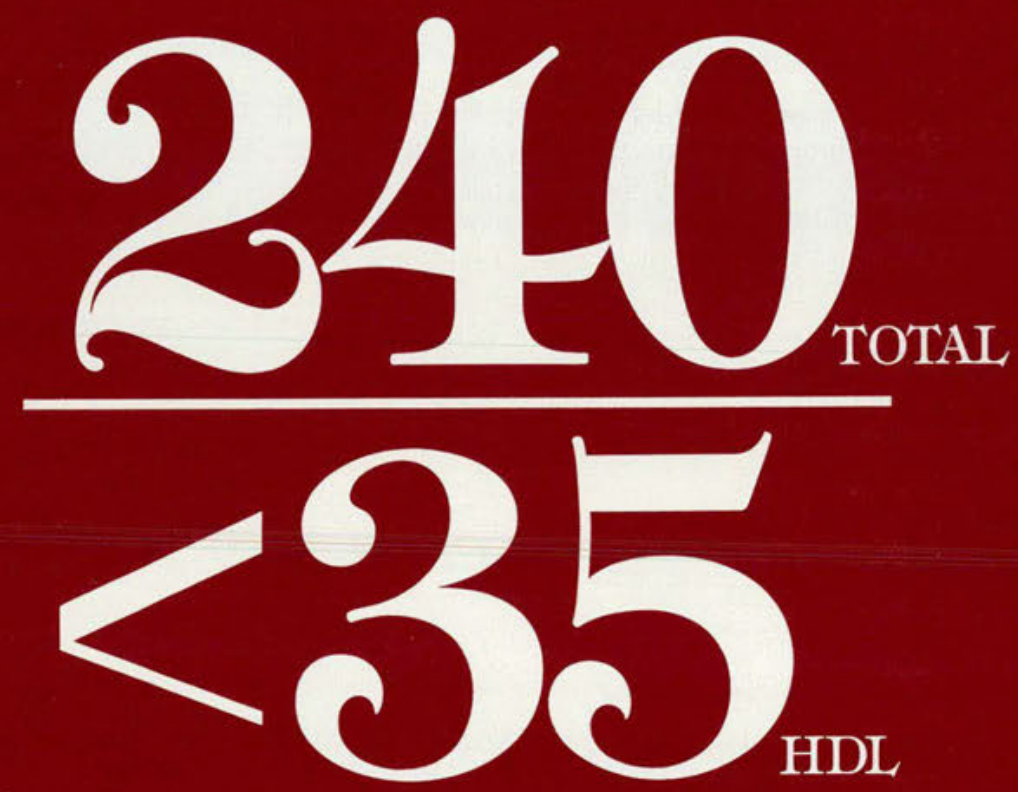

\section{Low HDL with elevated LDL and triglycerides: A common denominator of many heart attack victims}




\section{A powerful case for (D) DD) (gemfibrozil) ${ }_{\text {Tablets }}^{\text {600m }}$}

LOPID is indicated for reducing the risk of coronary heart disease in type Ilb patients with low HDL, in addition to elevated LDL and triglycerides, and who have had an inadequate response to weight loss, diet, exercise, and other pharmacologic agents such as bile acid sequestrants and nicotinic acid. LOPID is not indicated for the treatment of patients with low HDL cholesterol as their only lipid abnormality.

\section{Reduced heart attack incidence up to $62 \%$}

-in Helsinki Heart Study patients whose baseline HDL was $<35 \mathrm{mg} / \mathrm{dL}$ and median baseline LDL was $186 \mathrm{mg} / \mathrm{dL}$.' Incidence of serious coronary events was similar for LOPID and placebo subgroups with baseline HDL above the median $(46.4 \mathrm{mg} / \mathrm{dL}){ }^{1}$

\section{Raised low HDL 25\%}

-in these Helskinki Heart Study patients.

\section{RAISES HDL, LOWERS LDL AND TRIGLYCERIDES DRAMATICALLY REDUCES HEART ATTACK}

Contraindicated in patients with hepatic or severe renal dysfunction, including primary biliary cirrhosis, preexisting gallbladder disease, or hypersensitivity to gemfibrozil. LOPID may increase cholesterol secretion into the bile, leading to cholelithiasis. Caution should be exercised when anticoagulants are given in conjunction with LOPID. *Defined as a combination of definite coronary death and/or definite myocardial infarction. $P=.013 ; 95 \% \mathrm{Cl} 13.3$ to 111.5 .

Reference 1. Data on file, Medical Affairs Dept, Parke-Davis.

Please see last page of this advertisement for warnings, contraindications, and brief summary of prescribing information. 
WARNINGS. 1. Because of chemical, pharmacological, and clinical similarities be tween gemfibrozil and clofibrate, the adverse findings with clofibrate in two large clinic studies may also apply to gemfibrozil. In the first of those studies, the Coronary Drug Project, 1000 subjects with previous myocardial infarction were treated for five years with clofibrate. There was no difference in mortality between the clofibrate-treated subjects and 3000 placebo-treated subjects, but twice as many clofibrate-treated subjects developed cholelithiasis and cholecystitis requiring surgery. In the other study, conducted by the World Health Organization (WHO), 5000 subjects without known coronary heart disease were treated with clofibrate for five years and followed one year beyond. There was a statistically significant, $29 \%$, higher total mortality in the clofibrate treated than in a comparable placebo-treated control group. The excess mortality was due to a $33 \%$ increase in noncardiovascular causes, including malignancy, post cholecystectomy complications, and pancreatitis. The higher risk of clofibrate-treated subjects for gallbladder disease was confirmed.

During the Helsinki Heart Study and in the $11 / 2$ year follow-up period since the trial was completed, mortality from any cause was $59(2.9 \%)$ in the Lopid group and 55 $(2.7 \%)$ in the placebo group. Mortality from any cause during the double-blind portion of the study was 44 deaths in the Lopid group and 43 in the placebo group. Because o the more limited size of the Helsinki Heart Study, this result is not statistically significantly different from the $29 \%$ excess mortality seen in the clofibrate group in the separate WHO study. Noncoronary heart disease related mortality showed a $58 \%$ greater trend in the Lopid group ( 43 vs 27 patients in the placebo group, $p=0.056$ ). In the Helsinki Heart Study, the incidence of total malignancies discovered during the trial and in the $11 / 2$ years since the trial was completed was 39 in the Lopid group and 29 in the placebo group (difference not statistically significant). This includes 5 basal cell carcinomas in the Lopid group and none in the placebo group $(p=0.06$; historical data predicted an expected 4.7 cases in the placebo group). GI malignancies and deaths from malignancies were not statistically different between Lopid and placebo sub groups. Follow-up of the Helsinki Heart Study participants will provide further information on cause-specific mortality and cancer morbidity

2. A gallstone prevalence substudy of 450 Helsinki Heart Study participants showed a trend toward a greater prevalence of gallstones during the study within the Lopid treatment group $7.5 \%$ vs $4.9 \%$ for the place bo group, a $55 \%$ excess for the gemfibrozil group). A trend toward a greater incidence of gallbladder surgery was observed for the Lopid group (17 vs 11 subjects, a 54\% ex cess). This result did not differ statistically

from the increased incidence of cholecystectomy observed in the WHO study in the group treated with clofibrate. Both clofibrate and gemfibrozil may increase cholestero excretion into the bile leading to cholelithiasis. If cholelithiasis is suspected, gallbladder studies are indicated. Lopid therapy should be discontinued if gallstones are found.

3. Since a reduction of mortality from coronary artery disease has not been demonstrated and because liver and interstitial cell testicular fumors were increased in rats, Lopid should be administered only to those patients described in the INDICATIONS AND USAGE section. If a significant serum lipid response is not obtained, Lopid should be discontinued.

4 Concomitant Anticoagulants - Caution should be exercised when anticoagulants are given in conjunction with Lopid. The dosage of the anticoagulant should be reduced to maintain the prothrombin time at the desired level to prevent bleeding complications. Frequent prothrombin determinations are advisable until it has been definitely determined that the prothrombin level has stabilized.

5. Concomitant therapy with Lopid and Mevacor* (lovastatin) has been associated with rhabdomyolysis, markedly elevated creatine kinase (CK) levels and myoglobinuria. leading in a high proportion of cases to acute renal failure. In most subjects who have had an unsatisfactory lipid response to either drug alone, the possible benefit of combined therapy with lovastatin and gemfibrozil does not outweigh the risks of severe myopathy, rhabdomyolysis, and acute renal failure (See Drug Interactions). The use of fibrates alone, including Lopid, may occasionally be associated with myositis. Patients receiving Lopid and complaining of muscle pain, tenderness, or weakness should have prompt medical evaluation for myositis, including serum creatine kinase level deterr

myositis is suspected or diagnosed. Lopid therapy should be withdrawn.

6. Cataracts - Subcapsular bilateral cataracts occurred in $10 \%$, and unilateral in $6.3 \%$ of male rats treated with gemfibrozil at 10 times the human dose

PRECAUTIONS. 1. Initial Therapy - Laboratory studies should be done to ascertain that the lipid levels are consistently abnormal. Before instituting Lopid therapy, every attempt should be made to control serum lipids with appropriate diet, exercise, weight loss n obese patients, and control of any medical problems such as diabetes mellitus and hypothyroidism that are contributing to the lipid abnormalities

Continued Therapy-Periodic determination of serum lipids should be obtained. and the drug withdrawn if lipid response is inadequate after 3 months of therapy

3. Drug Interactions - (A) Lovastatin: Rhabdomyolysis has occurred with combine gemfibrozil and lovastatin therapy. It may be seen as early as 3 weeks after initiation of combined therapy or after several months. In most subjects who have had an unsatisfactory lipid response to either drug alone, the possible benefit of combined therapy with lovastatin and gemfibrozil does not outweigh the risks of severe myopathy, rhab. domyolysis, and acute renal failure. There is no assurance that periodic monitoring of

(B) Anticoagulants: CAUTION SHOULD BE EXERCISED WHEN ANTICOAGU ANTS ARE GIVEN IN CONJUNCTION WITH LOPID. THE DOSAGE OF THE ANT COAGULANT SHOULD BE REDUCED TO MAINTAIN THE PROTHROMBIN TIME AT THE DESIRED LEVEL TO PREVENT BLEEDING COMPLICATIONS. FREQUEN PROTHROMBIN DETERMINATIONS ARE ADVISABLE UNTIL IT HAS BEEN

DEFINITELY DETERMINED THAT THE PROTHROMBIN LEVEL HAS STABILIZED

4. Carcinogenesis, Mutagenesis, Impairment of Fertility - Long-term studies have been conducted in rats and mice at one and ten times the human dose. The inc:dence of benign liver nodules and liver carcinomas was significantly increased in high dose male rats. The incidence of liver carcinomas increased also in low dose males, but this increase was not statistically significant $(p=0.1)$. In high dose female rats, there was a significant increase in the combined incidence of benign, and malignant liver neoplasms. In male and female mice, there were no statistically significant differences from controls in the incidence of liver tumors, shown to be carcinogenic with other fibrates.

Male rats had a dose-related and statistically significant increase of benign Leydig cell tumors at 1 and 10 times the human dose.

Electron microscopy studies have demonstrated a florid hepatic peroxisome prolifera tion following Lopid administration to the male rat. An adequate study to test for peroxsome proliferation has not been done in humans but changes in peroxisome morphology have been observed. Peroxisome proliferation has been shown to occur in humans with either of two other drugs of the fibrate class when liver biopsies were compared before and after treatment in the same individual.

Administration of approximately three or ten times the human dose to male rats for 10 weeks resulted in a dose-related decrease of fertility. Subsequent studies demonstrated that this effect was reversed after a drug-free period of about eight weeks, and it was not transmit ted to the offspring.

Pregnancy Category B-Reproduction studies have been performed in the rat at doses 3 and 9 times the human dose, and in the rabbit at 2 and 6.7 times the human dose. These studies have revealed no evidence of impaired fertility in females or harm to the fetus due to Lopid. Minor fetotoxicity was manifested by reduced birth rates observed at the high dose levels. No significant malformations were found among almost $400 \mathrm{off}$ spring from 36 litters of rats and 100 fetuses from 22 litters of rabbits.

There are no studies in pregnant women. In view of the fact that Lopid is tumorigenic in male and female rats, the use of Lopid in pregnancy should be reserved for those paients where the benefit clearly outweighs the possible risk to the patient or fetus.

6. Nursing Mothers - Because of the potential for tumorigenicity shown for gem fibrozil in rats, a decision should be made whether to discontinue nursing or discontinue the drug, taking into account the importance of the drug to the mother.

Hematologic Changes - Mild hemoglobin, hematocrit and white blood cell focreases have been observed in occasional patients following initiation of Lopid therapy. However, these levels stabilize during long-term administration. Rarely, severe anemia, leukopenia, thrombocytopenia, and bone marrow hypoplasia have been reported. Therefore, periodic blood counts are recommended during the first 12 months of Lopid administration.

8. Liver Function - Abnormal liver function tests have been observed occasionally during Lopid administration, including eleva. tions of AST (SGOT), ALT (SGPT), LDH, bilirubin, and alkaline phosphatase. These are usually reversible when Lopid is discontinued. Therefore periodic liver function studies are recommended and Lopid therapy should be terminated if abnormalities persist.

9. Use in Children - Safety and efficacy in children have not been established. ADVERSE REACTIONS. In the double-blind controlled phase of the Helsinki Heart Study, (t) 5 years. following adverse reactions were statistically more frequent in subjects in the Lopid group (placebo incidence in pare (23.8\%); dyspepsia. $19.6 \%$ (11.9\%); abdominal pain, $9.8 \%$ (5.6\%); acute appendicitis (histologically confirmed in most cases where data are available), $1.2 \%(0.6 \%)$; atrial fibrillation, $0.7 \%(0.1 \%)$

Adverse events reported by more than $1 \%$ of subjects, but without a significant difference between groups (placebo incidence in parentheses) were: diarrhea, 7.2\% (6.5\%); fatigue, $3.8 \%$ (3.5\%); nausea/vomiting. $2.5 \%(2.1 \%)$; eczema, $1.9 \%(1.2 \%)$; rash Gallbladder surgery was performed in $0.9 \%$ of Lopid and $0.5 \%$ of placebo subjects, a $64 \%$ excess, which is not statistically different from the excess of gallbladder surgery $64 \%$ excess, which is not statistically different from the excess of gallbladder
observed in the clofibrate compared to the placebo group of the WHO study

Nervous system and special senses adverse reactions were more common in the Lopid group. These included hypesthesia, paresthesias, and taste perversion. Other adverse reactions that were more common among Lopid treatment group subjects but where a causal relationship was not established include cataracts, peripheral vascular disease, and intracerebral hemorrhage

From other studies it seems probable that Lopid is causally related to the occurrence
musculoskeletal symptoms (See WARNINGS), and to abnormal liver function tests and hematologic changes (See PRECAUTIONS)

Reports of viral and bacterial infections (common cold, cough, urinary tract infections) were more common in gemfibrozil-treated patients in other controlled clinical trials of 805 patients. Additional adverse reactions that have been reported for gemfibrozil are listed below by system. These are categorized according to whether a causal relationship to treatment with Lopid is probable or not established:

CAUSAL RELATIONSHIP PROBABLE: Gastrointestinal: cholestatic jaundice; Centra/ Nervous System dizziness, somnolence, paresthesia, peripheral neuritis, decreased libido, depression, headache; Eye: blurred vision; Genitourinary: impotence: Musculoskeletal: myopathy, myasthenia, myalgia, painful extremities, arthralgia, synovitis, rhabdomyolysis (see WARNINGS and Drug Interactions under PRECAU. TIONS); Clinical Laboratory: increased creatine phosphokinase, increased bilirubin, in creased liver transaminases (AST [SGOT], ALT [SGPT]), increased alkaline phosphatase; Hematopoietic: anemia, leukopenia, bone marrow hypoplasia, eosinophilia; Im. munologic: angioedema, laryngeal edema, urticaria; Integumentary: exfoliative dermatitis, rash, dermatitis, pruritus

CAUSAL RELATIONSHIP NOT ESTABLISHED: General: weight loss; Cardiac: extrasys toles; Gastrointestinal: pancreatitis, hepatoma, colitis; Central Nervous System: confusion, convulsions, syncope; Eye: retinal edema: Genitourinary: decreased male fertility: Clinical Laboratory: positive antinuclear antibody; Hematopoietic: thrombocytopenia; Immunologic: anaphylaxis, Lupus-like syndrome, vasculitis; Integumentary: alopecia DOSAGE AND ADMINISTRATION. The recommended dose for adults is $1200 \mathrm{mg}$ MANAGEMENT OF OVERDOSE. While there has been no reported case of over. dosa symptomatic supportive measures should be taken should it occur. References: 1. Frick MH, Elo O. Haapa K, et al: Helsinki Heart Study: Primary preven tion trial with gemfibrozil in middle-aged men with dyslipidemia. N Engl J Med 1987:317:1237-1245. 2. Manninen V, Elo O, Frick MH, et al: Lipid alterations and decline in the incidence of coronary heart disease in the Helsinki Heart Study. JAMA 1988; 260:641-651.3. Nikkila EA: Familial lipoprotein lipase deficiency and related disorders of chylomicron metabolism. In Stanbury J. B. et al. (eds.): The Metabolic Basis of Inherited ed. McGraw-Hill, 1983 , Chap 30 pp 622.642 .

Caution - Federal law prohibits dispensing without prescription. 
occurred only in the CWT group and not in the aerobic exercise group (Table 3 ). Although the final amount of cumulative weight lifted in the CWT group ( $877 \pm 22 \mathrm{lb})$ was about 100 lb greater than that in the aerobic exercise group $(780 \pm 45 \mathrm{lb})$, these values were not significantly different at the .05 level of probability. The use of the cycle, resulting in simultaneous dynamic exercise of the arms and legs, as well as the possible performance of other activities away from the rehabilitation program, may have contributed to an increase in upper body strength in some subjects in the aerobic exercise group. However, the change in strength in the CWT group $(157 \pm 8 \mathrm{lb})$ showed much less variance compared with the change seen in the aerobic exercise group $(94 \pm 46 \mathrm{lb})$, a result suggesting that the addition of CWT was much more likely to produce the desired improvements in upper body strength.

\section{Comment}

The use of weight-training exercises in cardiac rehabilitation has been gaining in acceptance. Our findings suggest that stable cardiac patients can safely perform a coordinated program of CWT and aerobic exercise as early as 6 weeks into phase 2 cardiac rehabilitation with resultant improvements in both strength and aerobic capacity. Further studies incorporating more patients with three-vessel coronary artery disease will need to be conducted before these results can be generalized to all patients in phase 2 cardiac rehabilitation programs.

\section{Acknowledgment}

Thanks are expressed to William H. Beierwaltes, $P h D$, and Karen R. Kalbfleisch, $P h D$, for their helpful suggestions in the preparation of the manuscript, and Joseph C. Rogers, DO, for his support and guidance of this project.

\section{References}

1. Vander LB, Franklin BA, Wrisley D, et al: Acute cardiovascular responses to Nautilus exercise in cardiac patients: Implications for exercise training. Ann Sports Med 1986;2:165-169. 2. Kelemen MH, Stewart KJ, Gillilan RE, et al: Circuit weight training in cardiac patients. $J$ Am Coll Cardiol 1986; 7:38-42.

3. Butler RM, Beierwaltes WH, Rogers FJ: The cardiovascular response to circuit weight training in patients with cardiac disease. J Cardiopulmonary Rehabil 1987;7:402-409.

4. Haslam DRS, McCartney N, McKelvie RS, et al: Direct measurements of arterial blood pressure during formal weightlifting in cardiac patients. J Cardiopulmonary Rehabil 1988;8:213225

5. Gettman LR, Pollock ML: Circuit weight training: A critical review of its physiological benefits. Physician Sportsmed $1981 ; 9: 44-60$.

6. Stewart KJ, Mason M, Kelemen MH: Three-year participation in circuit weight training improves muscular strength and self-efficacy in cardiac patients. $J$ Cardiopulmonary Rehabil 1988;8:292-296.

7. Sparling PB, Cantwell JD, Dolan CM, et al: Strength training in a cardiac rehabilitation program: A six-month follow-up. Arch Phys Med Rehabil 1990;71:148-152.

8. Sparling PB, Cantwell JD: Strength training guidelines for cardiac patients. Physician Sportsmed 1989;17:190-196.

9. Kelemen MH: Resistive training safety and assessment guidelines for cardiac and coronary prone patients. Med Sci Sports Exerc 1989;21:675-677.

10. American College of Physicians Health and Public Policy Committee: Cardiac rehabilitation services. Ann Intern Med 1988;109:671-673.

11. Bruce RA: Exercise testing of patients with coronary heart disease: Principles and normal standards for evaluation. Ann Clin Res 1971;3:323-332.

12. Kent KM, Bentivoglio LG, Block PC, et al: Long-term efficacy of percutaneous transluminal coronary angioplasty (PTCA): Report from the National Heart, Lung, and Blood Institute PTCA Registry. Am J Cardiol 1984;53:27C-31C.

13. MacDougall JD, Tuxen D, Sale DG, et al: Arterial blood pressure response to heavy resistance exercise. $J$ Appl Physiol 1985;58:785-790.

14. Nelson RR, Gobel FL, Jorgensen CR, et al: Hemodynamic predictors of myocardial oxygen consumption during static and dynamic exercise. Circulation 1974;50:1179-1189.

15. Gettman LR, Ward P, Hagan RD: A comparison of combined running and weight training with circuit weight training. Med Sci Sports Exerc 1982;14:229-234.

16. Gettman LR, Ayres JJ, Pollock ML, et al: The effect of circuit weight training on strength, cardiorespiratory function, and body composition of adult men. Med Sci Sports 1978;10:171-176. 17. Gettman LR, Ayres JJ, Pollock ML, et al: Physiologic effects on adult men of circuit strength training and jogging. Arch Phys Med Rehabil 1979;60:115-120.

18. Crozier Ghilarducci LE, Holly RG, Amsterdam EA: Effects of high resistance training in coronary artery disease. Am J Cardiol 1989;64:866-870. 